\title{
XXV. True apparent right ascension of Dr. Maskelyne's 36 stars for every day in the year 1821
}

\section{Rev. J. Grooby}

To cite this article: Rev. J. Grooby (1821) XXV. True apparent right ascension of Dr. Maskelyne's 36 stars for every day in the year 1821, Philosophical Magazine Series 1, 58:280, 110-112, DOI: $10.1080 / 14786442108652581$

To link to this article: http://dx.doi.org/10.1080/14786442108652581

$$
\text { 册 Published online: } 29 \text { Jul } 2009 .
$$

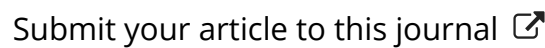

Џ Article views: 2 
at one-third, 3 , at one-fourth, 4, 8c. Also at two-thirds the length is marked $1 \frac{1}{2}$, at two-fifths, $2 \frac{1}{2}, 8 c$. And $s 0$ of all the fractions, sufficiently minutely. These numbers extend as high as the specific gravity of platina;-the pivot of the instrument represents unity, and a notch is made at the further end.

In using this instrument, any convenient weight is suspended by a hook from the notch at the end of the scale. The body under examination is to be suspended to the other end by a horsehair, and slid along till an equilibrium is produced. It is then, without altering its situation on the beam, to be immersed in water, and balanced a second time by sliding the weight. The hook of the latter then marks the specific gravity on the scale.

The demonstration of this is very simple. 'The instrument' being supposed in equilibrium, and $\mathrm{BD}$ (see figure) and the weight of the counterpoise being constant, the weight of the body varies as the distance of the counterpoise from $B$, by the common principle of the lever. Hence, if $\mathrm{C}$ be the place of the weight at the conclusion of the operation,

Weight in water : weight in air : : B C : B A. And, by subtraction, the loss of weight in water : weight in air : : AC:AB; and hence

$\frac{\text { wt. in air }}{\text { loss }}=\frac{A B}{A C}=$ the spec. grav.; which is the rule. a. E. D.

Substances lighter than water may have, if necessary, their specific gravity ascertained by the usual method; a scale of equal parts being cut on the opposite side of the beam, and the article to be weighed placed in a notch for the purpose. For mineralogy, however, this will seldom be necessary. The bottom of the notch A (at the sinaller end) should be in a line with the edge of the scale, its sides being a little raised. The top of the shorter end should be rather the thickest part of it, to allow the horse-hair, by which the mineral is suspended, to swing clear. This mode will be found very delicate and accurate, and a hook must not be used, as it cannot be balanced.

The instrument, in this form, is exceedingly compact, and may be reduced to a simple rod.

The principle is capable of being applied (as in an instrument I have made) to an arc of a circle, with a rod resembling in its application a common bent lever.

XXV. True apparent Right Ascension of Dr. Maskelyne's 36 Stars for every Day in the Year 1821. By the Rev. J. Groosx.

[Continued from p. 52.] 
True apparent Right Ascension of Dr. Maskelyne's 36 Stars.

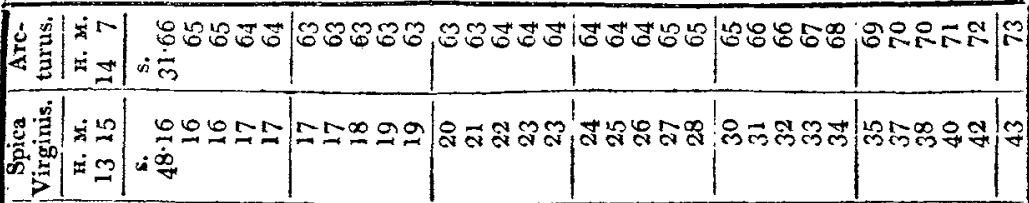

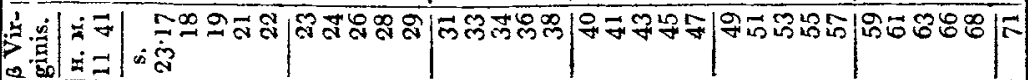

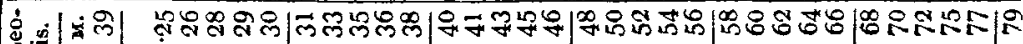

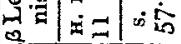

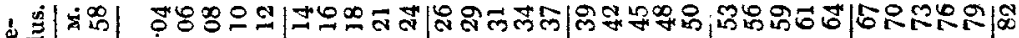

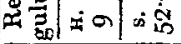

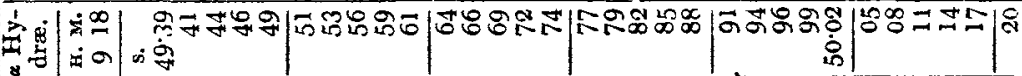

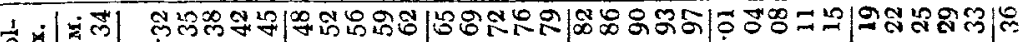

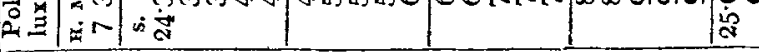

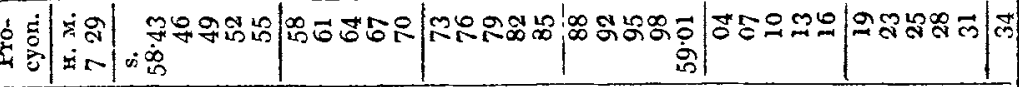

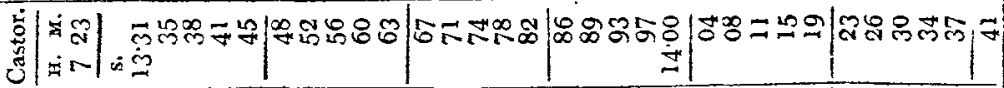

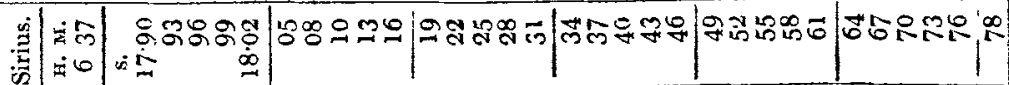

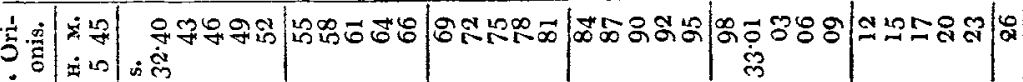

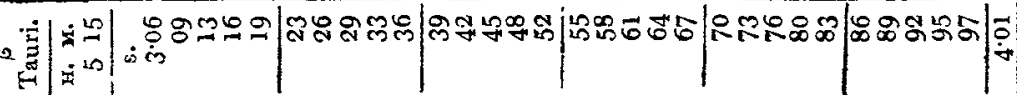
象| in

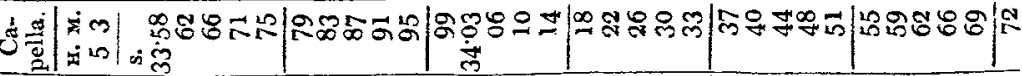

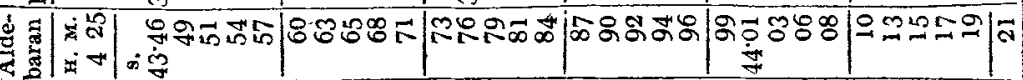

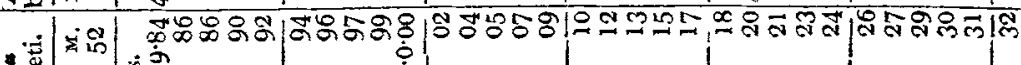
ज्ञ तो में

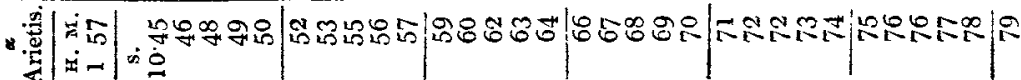

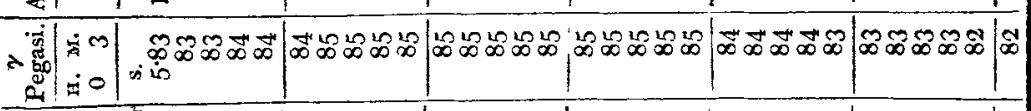

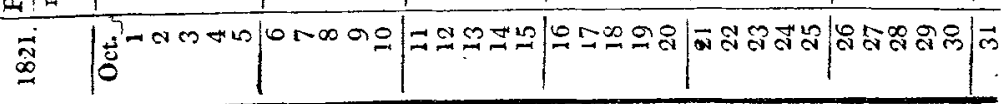




\section{True apparent Right Ascension of Dr. Maskelyne's 96 Stars.}

\begin{tabular}{|c|c|c|c|c|c|c|c|}
\hline $\mathbb{E}$ & $\begin{array}{l}\dot{4} 20 \\
\dot{4} 20\end{array}$ & 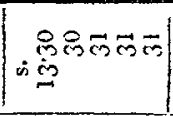 & 1 & & $5 \mathrm{~m}$ & 89 & $8 \pi$ \\
\hline & 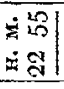 & कूু & 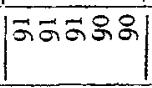 & $\infty$ & $\infty$ & 8 & $25 \sqrt{5}$ \\
\hline & $\begin{array}{l}4 \\
4 \\
4\end{array}$ & 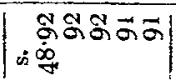 & $8 \infty$ & $\infty \infty$ & $\infty 2$ & $9 \mathbb{2}$ & 8818 \\
\hline & 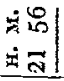 & 吾 988 & $\mid 58080$ & 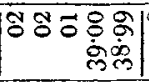 & \%5ร์ல & 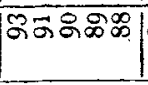 & $\mid+\infty \infty \infty \infty \infty \times \infty$ \\
\hline & 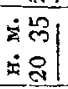 & 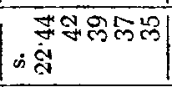 & लिकत्वां & $\pi 90 \pm N$ & 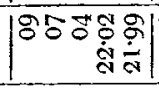 & $5 \infty$ & 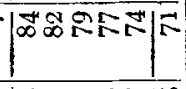 \\
\hline & $\begin{array}{l}\dot{z}^{\infty} \\
\dot{z}_{i}\end{array}$ & 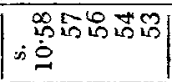 & 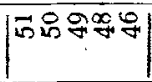 & अ 39 원이 & लिलेलिल & 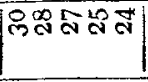 & ส $\approx \Omega \infty=\left.\right|^{20}$ \\
\hline$\approx$ & $\begin{array}{l}\dot{\pi}^{4} \\
\text { iे }\end{array}$ & 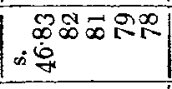 & |OORNMA & 109580 & 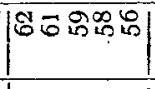 & 4 & +29619 \\
\hline & 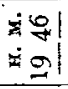 & 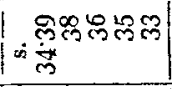 & 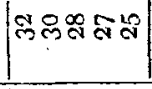 & 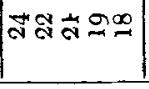 & 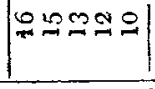 & $|85858|$ & 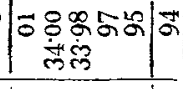 \\
\hline & 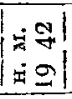 & कृ & 18:2ร8 & & & & 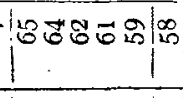 \\
\hline & 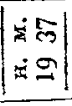 & 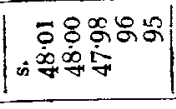 & | & $(\infty \vec{\infty} \infty \vec{\infty}$ & 吪卡 & 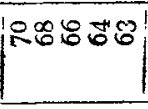 & 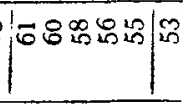 \\
\hline & 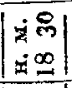 & 要8880 & \& & $B=$ & 10 & $=$ & 8 \\
\hline & 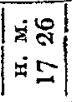 & $\begin{array}{l}=8588 \\
\text { की }\end{array}$ & 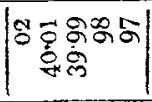 & 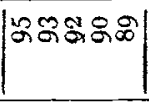 & $\left|\begin{array}{llll}\infty & \infty & \infty & \infty \\
\infty & \infty & \infty\end{array}\right|$ & W. & $|\operatorname{|rg} 2 \pi x|$ \\
\hline है: & 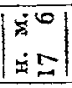 & 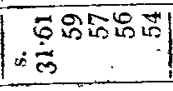 & & & 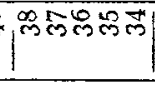 & লিল্লিন্র & $\mid \pi \quad$ a \\
\hline 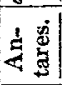 & 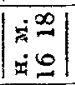 & 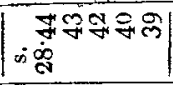 & 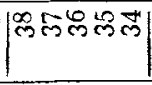 & 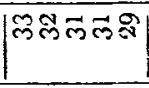 & 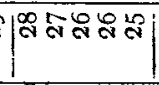 & โสखส & สสสลส สิ \\
\hline $\mid$ & 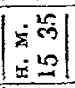 & 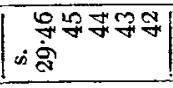 & | & 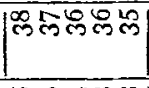 & 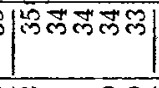 & नुलूल्लूल & 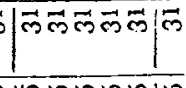 \\
\hline क्ष & 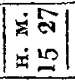 & क्लूल लूलूल & बి. & 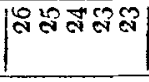 & |ีสสลำ & $10 \infty \approx 50$ & $\mid$ \\
\hline 造 & 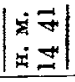 & 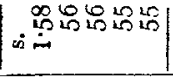 & 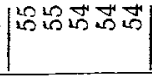 & 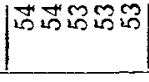 & : & 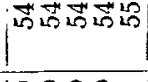 & 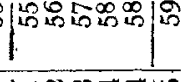 \\
\hline 8 & 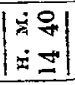 & ${ }_{\infty}^{2} \underset{\dot{0}}{\infty} \approx=$ & & 88 & 188889 & $9=$ & 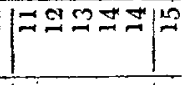 \\
\hline 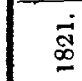 & & $\therefore-$ & & & å & எ & 官| \\
\hline
\end{tabular}

